\title{
Preisträgerinnen und Preisträger 2020
}

\section{WILHELM-CONRAD- RÖNTGEN-PREIS}

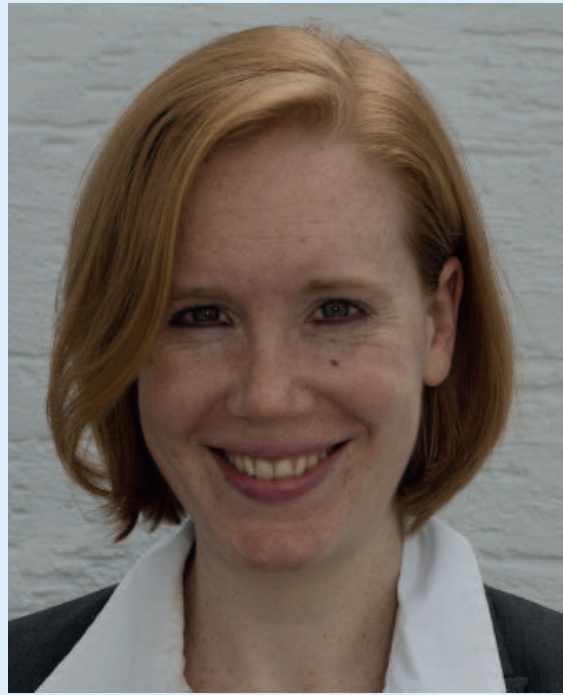

PD Dr. Bettina Baeßler, Zürich

Zum Gedenken an ihren Namensgeber vergibt die Deutsche Röntgengesellschaft seit 1979 jährlich den Wilhelm-Conrad-Röntgen-Preis.

PD Dr. Bettina Baeßler wurde für ihre wissenschaftliche Arbeit „Cardiac MRI and Texture Analysis of Myocardial T1 and T2 Maps in Myocarditis with Acute versus Chronic Symptoms of Heart Failure" ausgezeichnet.

\section{MARIE-CURIE-RING}

Der Marie-Curie-Ring zeichnet eine/n herausragende/n junge/n Wissenschaftler/ in der Radiologie aus. Grundlage der Begutachtung sind die gesamten bisherigen Leistungen, die im besonderen Maße befähigen sollen, eine herausragende wissenschaftliche Laufbahn zu vollenden.

PD Dr. Lars Schimmöller erhielt den MarieCurie-Ring in Anerkennung seiner herausragenden Leistungen auf dem Gebiet der Radiologie.

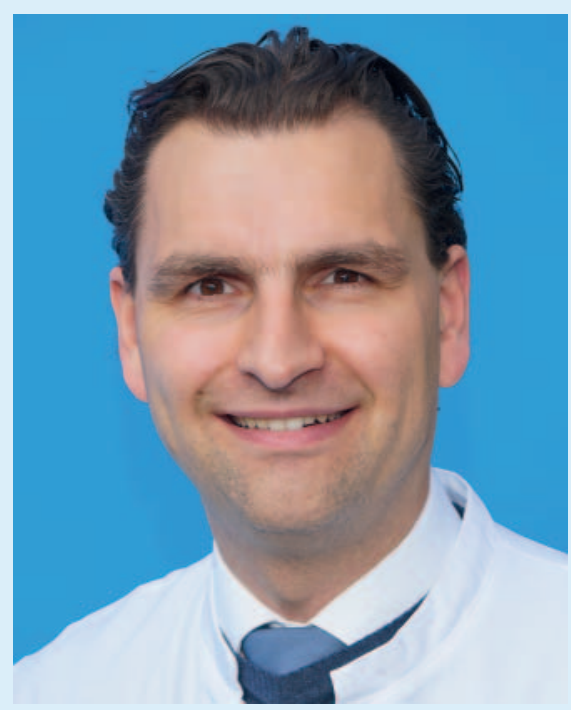

PD Dr. Lars Schimmöller, Düsseldorf

\section{WALTER-FRIEDRICH-PREIS}

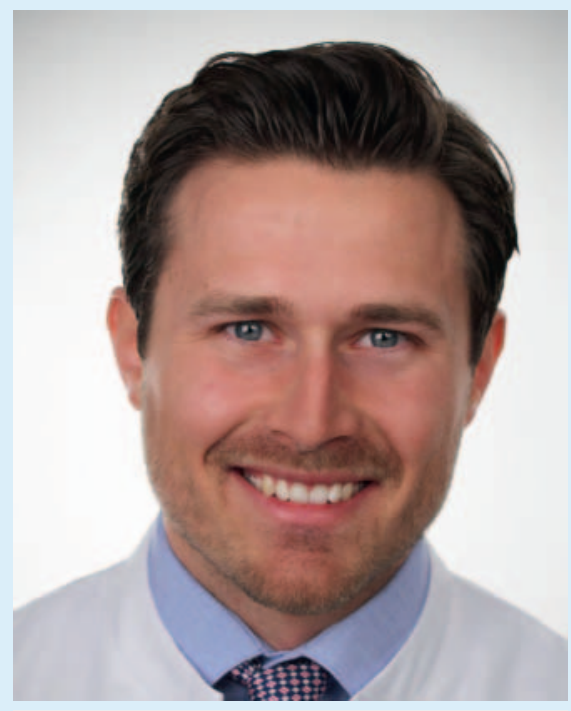

Dr. Daniel Paech, Heidelberg

Der Walter-Friedrich-Preis wird jährlich verliehen für herausragende wissenschaftliche Arbeiten, für beispielgebende Publikationen und Initiativen zur Verbesserung der Lehre und für vorbildliche praktische Ergebnisse in der Medizinischen Radiologie inklusive technischer Entwicklungen. Er berücksichtigt in besonderer Weise die Gemeinsamkeit von Physik, Technik, Biologie und Medizinischer Radiologie.
Dr. Daniel Paech wurde für die wissenschaftliche Arbeit „Metabolische Bildgebungstechniken am 7-Tesla-Ultra-Hochfeld-MRT bei neuroonkologischen Erkrankungen“ ausgezeichnet.

\section{PROMOTIONSPREIS DER DRG}

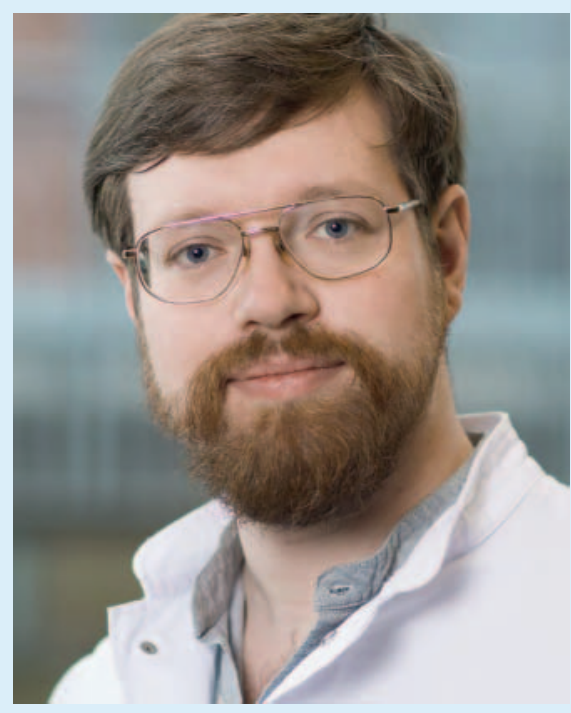

Dr. Manuel Kolb, Tübingen

Der Promotionspreis der Deutschen Röntgengesellschaft wurde erstmals im Jahr 2012 ausgeschrieben, um wissenschaftlich engagierte Studierende und Mediziner/ innen zu motivieren und zu fördern. Im Fall einer Auszeichnung der eingereichten $\mathrm{Ar}$ beit wird diese als Originalarbeit in der Fachzeitschrift RöFo veröffentlicht.

Dr. Manuel Kolb erhielt den Preis für seine wissenschaftliche Arbeit „Funktionelle Magnetresonanztomografie der akuten Nierentransplantatabstoßung im experimentellen Tiermodell“.

\section{YOUNG INVESTIGATOR}

\section{AWARD}

Seit dem Jahr 2009 werden alljährlich anlässlich des Deutschen Röntgenkongresses herausragende Arbeiten und Kongressbeiträge junger Wissenschaftler/innen mit dem Young Investigator Award (YIA) ge- 


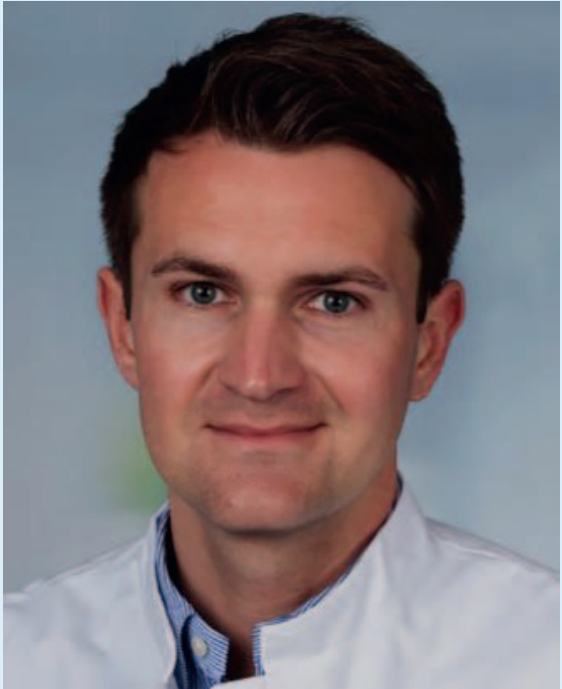

Dr. Alexander Isaak, Bonn

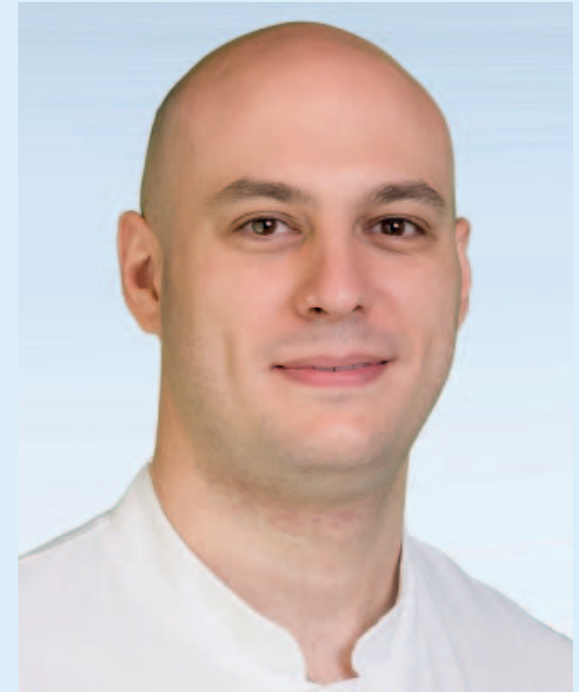

Dr. Federico Pedersoli, Aachen ehrt. Mit dem YIA sollen die Leistungen des wissenschaftlichen Nachwuchses in der Bildgebung herausgehoben und gefördert werden.

Dr. Alexander Isaak erhielt den Young Investigator Award für seine wissenschaftliche Arbeit „Charakterisierung der zirrhotischen Kardiomyopathie und deren Assoziation mit dem Schweregrad der Lebererkrankung: Eine prospektive multiparametrische kardiale und hepatische MRT-Studie" und

Dr. Federico Pedersoli für seine Arbeit „Vergleich der Effektivität der Hypertrophieinduktion nach unilobärer Y90-Radioembolisation vs. Pfortaderembolisation: Eine prospektive Tierstudie“.

\section{WACHSMANN-INNOVATIONSPREIS}

Der Wachsmann-Innovationspreis wird in Anerkennung der engagierten Weiterentwicklung und erfolgreichen Implementierung von zentralen Angeboten, Leistungen und Programmen der Deutschen Röntgengesellschaft verliehen.

2020 wurde mit dem Preis die Arbeitsgruppe „unirad/CoRad-19“ gewürdigt. Ihre Mitglieder haben interaktive E-Learning-Module für Medizinstudierende entwickelt und auf der Lernplattform conrad für die universitäre Lehre zur Verfügung gestellt. Als unmittelbare Antwort auf die Corona-Pandemie erstellten sie in kürzester Zeit ein „Notfallpaket“, welches das gesamte Spektrum der radiologischen Ausbildung abdeckt.

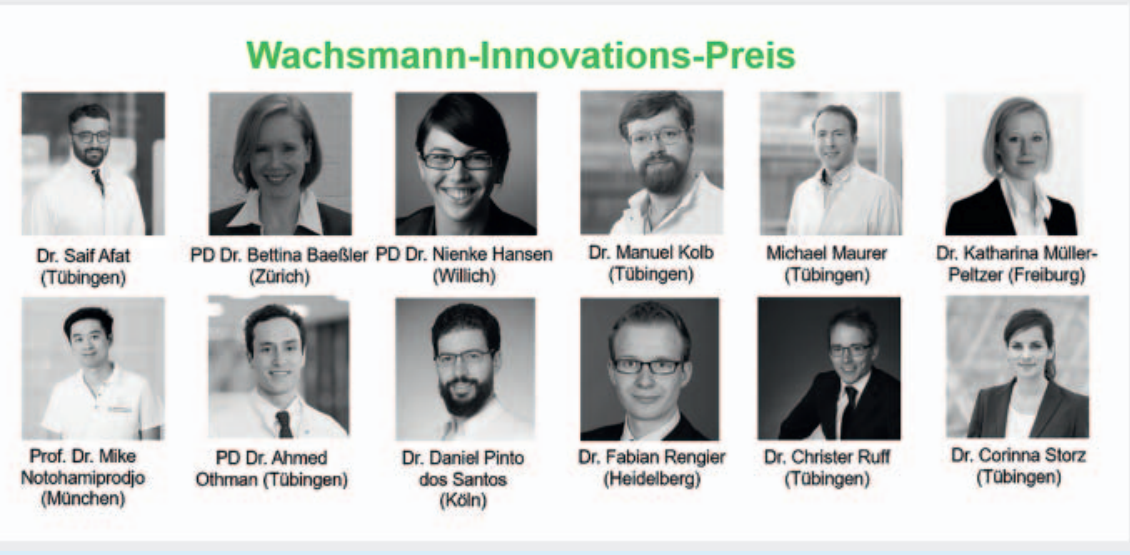

Arbeitsgruppe unirad/CoRad-19 
Zudem erhielt eine zweite Preisträgergruppe den Wachsmann-Innovationspreis im Jahr 2020: Prof. Dr. Martin Mack gemeinsam mit der DRG-Geschäftsstelle. Sie trugen maßgeblich dazu bei, dass innerhalb kürzester Zeit der 101. Deutsche Röntgenkongress 2020 in ein digitales Format überführt werden konnte. Somit war auch in Pandemie-Zeiten eine hochwertige Fortbildung gewährleistet, ebenso wie eine überzeugende Plattform für den wissenschaftlichen Nachwuchs und die Industrie.

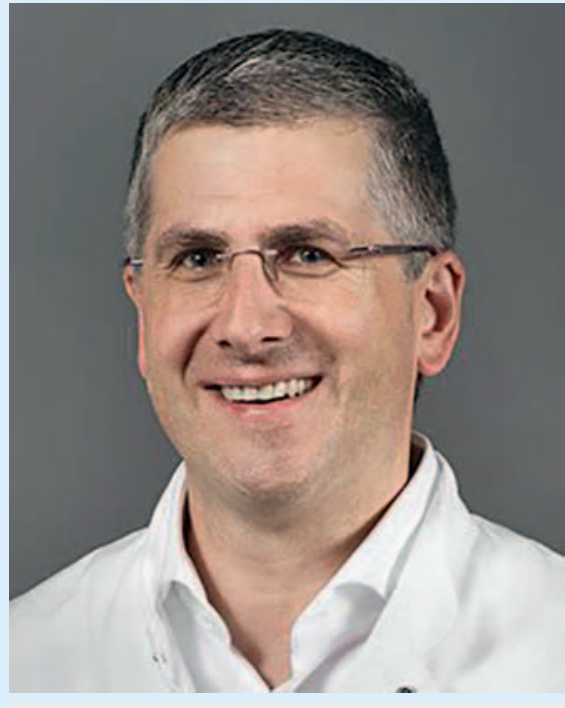

Prof. Dr. Martin Mack, München

\section{BESTER CASE REPORT}

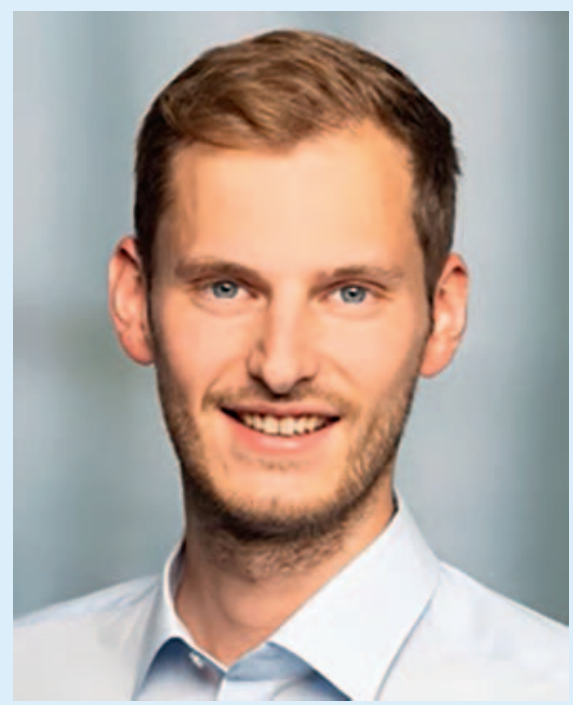

Das Forum Junge Radiologie vergab 2020 erstmalig den Preis für den besten Case Report, der im Rahmen des RÖKO DIGITAL vorgestellt wurde. Entscheidend war das Votum des Kongresspublikums.

Dr. Hauke Gerdes wurde für seinen Vortrag zu „Gallensteinileus als seltene Ursache eines Dünndarmileus“ ausgezeichnet.

Dr. Hauke Gerdes, Lübeck

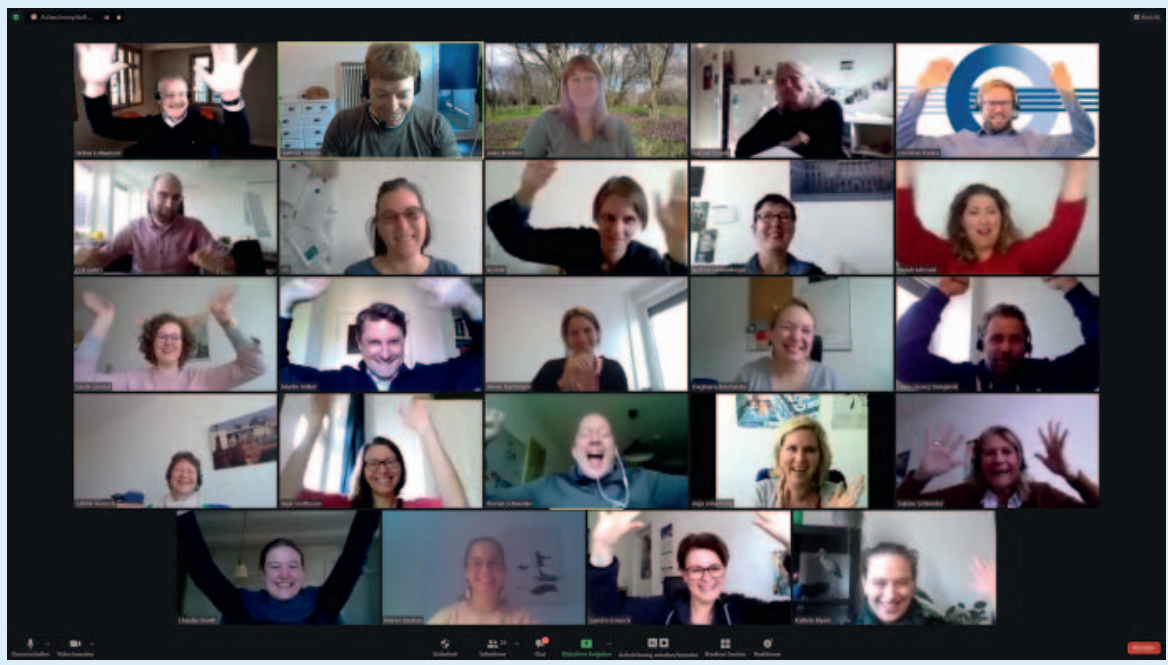

DRG-Geschäftsstelle, Berlin

Die Preisverleihungen im Rahmen von RÖKO DIGITAL können Sie hier als Video ansehen:

www.2020-digital.roentgenkongress.de > Allgemein > Die Preisträgerinnen und Preisträger 2020 